\title{
Ultraviolet Spectroscopy of Comet 9P/Tempel 1 with Alice/Rosetta during the Deep Impact Encounter
}

\author{
Paul D. Feldman \\ Department of Physics and Astronomy, The Johns Hopkins University \\ Charles and 34th Streets, Baltimore, MD 21218-2695 \\ pdf@pha. jhu.edu \\ S. Alan Stern, Andrew J. Steffl, Joel Wm. Parker \\ Space Science $\&$ Engineering Division, \\ Southwest Research Institute, 1050 Walnut Street, Suite 400, Boulder, CO 80302 \\ David C. Slater \\ Space Science $\&$ Engineering Division, \\ Southwest Research Institute, 6220 Culebra Road, San Antonio, TX 78238 \\ Michael F. A'Hearn \\ Department of Astronomy, University of Maryland, \\ College Park MD 20742-2421 \\ Jean-Loup Bertaux \\ Service d'Aéronomie du CNRS, BP 3, F-91371 Verrières-le-Buisson, France \\ Michel C. Festou ${ }^{1}$ \\ Observatoire Midi-Pyrénées, 14, avenue E. Belin, F-31400 Toulouse, France
}

\begin{abstract}
We report on spectroscopic observations of periodic comet 9P/Tempel 1 by the Alice ultraviolet spectrograph on the Rosetta spacecraft in conjunction with NASA's Deep Impact mission. Our objectives were to measure an increase in atomic and molecular emissions produced by the excavation of volatile sub-surface
\end{abstract}


material. We unambiguously detected atomic oxygen emission from the quiescent coma but no enhancement at the $10 \%(1-\sigma)$ level following the impact. We derive a quiescent $\mathrm{H}_{2} \mathrm{O}$ production rate of $9 \times 10^{27}$ molecules $\mathrm{s}^{-1}$ with an estimated uncertainty of $\sim 30 \%$. Our upper limits to the volatiles produced by the impact are consistent with other estimates.

Subject headings: comets: individual (9/P Tempel 1) — ultraviolet: solar system

\section{INTRODUCTION}

The Deep Impact mission (A'Hearn et al. 2005a) successfully placed a $364 \mathrm{~kg}$ impactor onto the surface of comet $9 \mathrm{P} /$ Tempel 1 at a relative velocity of $10.3 \mathrm{~km} \mathrm{~s}^{-1}$ on 2005 July 4 at 05:52:03 UT (as seen from Earth). The event was observed by cameras aboard the mother spacecraft (A'Hearn et al. 2005b) and by a large number of Earth- and space-based telescopes as part of an extensive campaign to study the comet prior to, during, and in the course of several days following the impact (Meech et al. 2005). Several instruments on board the Rosetta spacecraft, which is on a trajectory for a rendezvous with comet 67P/Churyumov-Gerasimenko in 2014, were programmed to observe comet Tempel 1 from June 28 through July 14, 2005. During this period Rosetta was located between 0.54 and $0.51 \mathrm{AU}$ from the comet, which was observed at a solar elongation angle varying from $93.4^{\circ}$ to $88.1^{\circ}$ and solar phase angle varying from $65.1^{\circ}$ to $72.5^{\circ}$. At the time of impact these parameters were $0.531 \mathrm{AU}, 91.1^{\circ}$, and $68.2^{\circ}$, respectively. Perihelion, at $1.506 \mathrm{AU}$, occurred on July 5. Imaging of the comet with the OSIRIS cameras on Rosetta has been reported by Küppers et al. (2005) and Keller et al. (2005).

Alice was the only space-based spectrograph operating at wavelengths below $2000 \AA$ at the time of Deep Impact. The spectral region spanned by Alice is rich is molecular and atomic emissions of the primary volatile components of the cometary coma. These include several band systems of $\mathrm{CO}$ and the strongest resonance transitions of the principal atomic species, $\mathrm{C}, \mathrm{H}, \mathrm{O}, \mathrm{N}$, and $\mathrm{S}$, that have been observed in many comets over the past 30 years using orbiting observatories and sounding rockets (e.g., Feldman et al. 2004). Alice is also sensitive to emissions of oxygen and carbon ions. All of these species were expected to show an increase as a result of the excavation of volatile sub-surface material by the impactor.

Because of the comet's relatively large distance from the Sun and its low quiescent activity level, characteristic of Jupiter-family comets, Alice was only able to unambiguously detect the quiescent coma in O I $\lambda 1304$, from which a quiescent water production rate of $\sim 9 \times 10^{27}$ molecules $\mathrm{s}^{-1}$ is deduced. H I Lyman- $\beta$ is also marginally detected above the 
interplanetary background. A slow secular decrease in the brightness of O I $\lambda 1304$ with time was detected but we did not see any significant increase (i.e., 10\%) as a result of the Deep Impact event. Our upper limits on the increase in these emissions (and those of other species as well) are consistent with other reports of $\mathrm{H}_{2} \mathrm{O}$ and $\mathrm{CO}$ production from the impact.

\section{INSTRUMENT}

Alice is a lightweight, low-power, imaging spectrograph optimized for in situ cometary far-ultraviolet (FUV) spectroscopy. It is designed to obtain spatially-resolved spectra in the 700-2050 $\AA$ spectral band with a spectral resolution between 8 and and $12 \AA$ for extended sources that fill its $0.05^{\circ} \times 6.0^{\circ}$ field-of-view. Alice employs an off-axis telescope feeding a 0.15-m normal incidence Rowland circle spectrograph with a concave holographic reflection grating. The imaging microchannel plate detector utilizes dual solar-blind opaque photocathodes ( $\mathrm{KBr}$ and $\mathrm{CsI}$ ) and employs a two-dimensional delay-line readout. Details are given by Slater et al. (2001). Following launch on March 2, 2004, and instrument commissioning, Alice observed comet C/2002 T7 (LINEAR) on April 30 and May 17, 2004. These measurements demonstrated the feasibility of remote ultraviolet observations from Rosetta, and served as a baseline for the data obtained during the Deep Impact campaign.

\section{OBSERVATION PROGRAM}

The Alice instrument observations during the Rosetta Deep Impact campaign consisted

of a repeated series of five "jailbar" exposures stepped across Tempel 1. Each step was shifted perpendicular to the slit length by $0.046^{\circ}$, which is slightly less than the slit width of $0.05^{\circ}$, providing some spatial overlap between steps. The five jailbar steps in each series therefore stepped the Alice boresight a total distance of $0.183^{\circ}$ centered on Tempel 1 . The exposure times were 26 minutes at the central step that pointed toward the target, and typically 19 minutes at the off-target positions. The total exposure time on the last offtarget point was 70 minutes due to the longer dwell time to accommodate observations by the MIRO instrument. This jailbar pattern was repeated almost continuously throughout the entire period from 2005 June 29 through July 14, except for three 3-hour interruptions for spacecraft reaction wheel offloads, and a few sets of dark exposures. At the time of the impact event, one set of jailbars was executed more quickly with exposure times of 9 minutes at each pointing except the last off-target point (coincident with the MIRO sub-millimeter boresight), which was 34 minutes. We then returned back to the original set of nominal exposure times for all subsequent jailbar observation sequences. 
A calibration star, $\rho$ Leo (B1 Ib), was observed on 2005 June 27 using the same jailbar pattern, though with different exposure times. Longer single-pointing exposures of the star were made for additional calibration and to check spacecraft pointing stability. The latter was checked by making exposures in time-tagged ("pixel-list") mode to look for variations in the count rate from the star. No variations were seen, implying the star was well-centered in the slit and that spacecraft pointing was accurate and stable relative to the width of the slit.

\section{SPECTRAL DATA}

The Alice slit consists of pixels that are $0.019^{\circ}$ wide in the spectral direction and $0.3^{\circ}$ high perpendicular to the dispersion. The width of the central region of the slit is $0.05^{\circ}$ which translates to a projected size of approximately $69,000 \times 416,000 \mathrm{~km}^{2}$ for a spatial pixel at the distance to the comet at the time of impact. Its large field-of-view, designed for in situ coma spectroscopy, makes Alice sensitive to the extended hydrogen and oxygen comae at the expense of emissions from the near-nucleus region. For each of the jailbar positions data are accumulated in two-dimensional arrays corresponding to wavelength and spatial axes. A data cube is produced by stacking these arrays with time as the third dimension. The data are displayed below as cuts along each of these three axes.

Inspection of the data cube shows that most of the ambient cometary emission occurs in the central row, row 15, of the spectral array. This row, integrated over time and divided into two time periods: pre- and post-impact, is shown in Fig. 1. Also shown are the spectra from rows 13 and 12, which are displaced $8.30 \times 10^{5} \mathrm{~km}$ and $1.24 \times 10^{6} \mathrm{~km}$ from the center of row 15 , respectively, projected on the sky. The background is a combination of detector dark counts and grating scattering of the interplanetary $\mathrm{H}$ I Lyman- $\alpha$ emission. The latter is qualitatively similar to the scattering function determined from pre-flight laboratory measurements (Slater et al. 2001). The photocathodes were deposited on the microchannel plate detector in such a way to leave a gap at Lyman- $\alpha$ to avoid saturation of the detector. This makes the absolute calibration at this wavelength uncertain and we will limit our discussion of hydrogen to Lyman- $\beta$.

The figure shows the clear detection of cometary O I $\lambda 1304$, together with a possible enhancement of the H I emissions above the interplanetary background. No other feature is detected. C I $\lambda 1657$, usually the brightest coma feature after Lyman- $\alpha$ and O I (Feldman et al. 2004), is not detected, but this may be in part due to the 3 times lower instrumental effective area at $1657 \AA$ compared with $1304 \AA$. In comet C/2002 T7 (LINEAR) on 2004 April 30 when the heliocentric velocity dependent O I and C I fluorescence efficiencies (evaluated 
at 1 AU) were similar to those for the Tempel 1 observations, the brightness of $\mathrm{C}$ I as observed by Alice was one-third that of O I. The absence of C I emission in Tempel 1 may reflect either a compositional difference or an effect of the different observation geometries for the two comets. There is no significant difference between the pre-impact and post-impact spectra. Upper limits to the expected C I and CO emissions are given in Table 1.

The spatial distributions of O I $\lambda 1304$ and H I Lyman- $\beta$ are shown in Fig. 2, again separated into temporal sums of the pre- and post-impact data. The $\mathrm{O}$ I is clearly associated with the comet and serves as a verification of the pointing of the spacecraft. Lyman- $\beta$, however, is seen to be a relatively minor enhancement to the interplanetary background. This is consistent with the coma modeling discussed below. The variation of the background Lyman- $\beta$ along the length of the slit is likely due to an incomplete flat-field characterization of the detector response. Unfortunately, there were no deep sky observations to characterize the interplanetary background, which had not been a problem for the 2004 comet observations. The general trend in the variation of Lyman- $\beta$ along the slit can be seen from an average of the two outer jailbar observations of $\rho$ Leo, as shown in Fig. 3 . The star was $\sim 23^{\circ}$ from the comet. While the trend is similar to that seen in Fig. 2, the star was sufficiently bright that its point spread function gave a significant contribution to rows 13-16 of the detector, even offset $0.09^{\circ}$ from the star, so it was not possible to use this profile to obtain a flat field that would enable us to extract the cometary Lyman- $\beta$ with any confidence.

The temporal variation of O I $\lambda 1304$, shown as a daily average, is given in Fig. 4 . There is no immediate increase following impact but this would not be expected as the lifetime against photodissociation of $\mathrm{H}_{2} \mathrm{O}$ at $1.506 \mathrm{AU}$ is about two days and that for $\mathrm{OH}$ is about four days. The high and low points seen on days 2 and 3 following impact, respectively, are less than $2-\sigma$ from the mean and are not statistically significant. There is also no indication, in the spectra obtained in the first few hours after impact, of any emission at the wavelengths of the strongest CO Fourth Positive bands, $1510 \AA(1,0)$, and $1478 \AA(2,0)$. The O I $\lambda 1304$ emission does show a slow secular decrease with time, and is consistent with the trend seen in the visible by Schleicher et al. (2006) and attributed to a slow decrease in the comet's quiescent outgassing rate.

\section{DISCUSSION}

\subsection{Quiescent Coma Models}

The $\mathrm{H}$ and $\mathrm{O}$ emissions can be modelled with a Haser model (see, e.g. Combi et al. 2004), in which we account for saturation of the solar flux with increasing column density 
along the line-of-sight through the coma. The model assumes radial outflow everywhere in the coma, which is valid for the case of the long Alice slit, and uses photodissociation rates appropriate to solar minimum from Table VI of Budzien et al. (1994). The mean outflow velocities used were $0.7 \mathrm{~km} \mathrm{~s}^{-1}$ for $\mathrm{H}_{2} \mathrm{O}, 19.6$ and $6.0 \mathrm{~km} \mathrm{~s}^{-1}$ for $\mathrm{H}$ (from $\mathrm{H}_{2} \mathrm{O}$ and $\mathrm{OH}$, respectively), and $1.2 \mathrm{~km} \mathrm{~s}^{-1}$ for O. Fluorescence efficiencies for O I are calculated following Dymond et al. (1989) using solar fluxes appropriate to solar minimum from Rottman et al. (2001), while for H I solar fluxes from SOHO/SUMER (Lemaire et al. 2002) are used. The spatial profiles for a model with an $\mathrm{H}_{2} \mathrm{O}$ production rate of $1 \times 10^{28}$ molecules s${ }^{-1}$, with the nucleus centered in row 15, are shown overplotted on the data of Fig. 2. The model is probably less reliable at distances beyond $10^{6} \mathrm{~km}$ from the nucleus because the loss of $\mathrm{H}$ and $\mathrm{O}$ by charge exchange with solar wind ions is not included in the model. Comparing the model with the integrated brightness in the 5 central rows leads to a derived quiescent production rate of $9 \times 10^{27}$ molecules $\mathrm{s}^{-1}$. The uncertainty in this result includes a $15 \%$ absolute calibration uncertainty and, more difficult to quantify, a $25 \%$ uncertainty in the parameters used in the Haser model.

This result may be compared with other estimates of the pre-impact $\mathrm{H}_{2} \mathrm{O}$ production rate. Küppers et al. (2005) using narrow-band $\mathrm{OH}$ images recorded by the OSIRIS wide-angle camera on board Rosetta, found a quiescent value $Q\left(\mathrm{H}_{2} \mathrm{O}\right)=(3.4 \pm 0.5) \times 10^{27}$ molecules s $^{-1}$, while Schleicher et al. (2006), from ground-based $\mathrm{OH}$ photometry, find $6 \times 10^{27}$ molecules s ${ }^{-1}$. Both of these use Haser models similar to the one described above to interpret the observed photodissociation product, $\mathrm{OH}$. Direct observations of water were made in both the infrared, using long-slit spectroscopy, by Mumma et al. (2005), and in the sub-millimeter, by the Odin satellite, observing at $557 \mathrm{GHz}$ (Biver et al. 2005). They report water production rates of $10.4 \times 10^{27}$ molecules $\mathrm{s}^{-1}$ and $(8.5 \pm 1.5) \times 10^{27}$ molecules $\mathrm{s}^{-1}$, respectively. Our result, within the uncertainties described above, are in general accord with the infrared and submm observations of $\mathrm{H}_{2} \mathrm{O}$.

\subsection{Gas Production Following Impact}

The upper limits on gas production derived from these data can then be compared with expectations based on other observations. For the amount of $\mathrm{H}_{2} \mathrm{O}$ produced by the impact, Küppers et al. give $(1.5 \pm 0.5) \times 10^{32}$ molecules and Biver et al. give $(1.4 \pm 0.35) \times$ $10^{32}$ molecules. Schleicher et al. give an upper limit of $4 \times 10^{32}$ molecules. Because their

long slit did not include all of the water produced by the impact, Mumma et al. only give an effective production rate, which is twice their quiescent value. 
Küppers et al. claim the total number of $\mathrm{H}_{2} \mathrm{O}$ molecules produced to be about $15 \%$ of the ambient number in the coma. We don't observe $\mathrm{H}_{2} \mathrm{O}$ but two of its dissociation products. In a time, $t$, the fraction of water dissociated into $\mathrm{H}$ and $\mathrm{OH}$ (and eventually $\mathrm{O}$ ) will be $\left(1-\exp \left(-t / \tau\left(\mathrm{H}_{2} \mathrm{O}\right)\right)\right.$, where $\tau\left(\mathrm{H}_{2} \mathrm{O}\right)$ at $1.506 \mathrm{AU}$ is $1.88 \times 10^{5} \mathrm{~s}$ for solar minimum conditions (Budzien et al. 1994). Thus, one day after impact we will see $\sim 35 \%$ of the $15 \%$ increase in the amount of $\mathrm{H}$, i.e., a $5 \%$ increase over ambient. With the Alice slit the increase will be even less as the fast moving $\mathrm{H}$ atoms $\left(\sim 20 \mathrm{~km} \mathrm{~s}^{-1}\right)$ will leave the area of the projected slit in a matter of hours. The increase in $\mathrm{O}$ will be even smaller as two photodissociations are required and the lifetime of $\mathrm{OH}$, the source of $90 \%$ of $\mathrm{O}$ in the coma (Combi et al. 2004), is about twice as long as that of $\mathrm{H}_{2} \mathrm{O}$. Promptly produced $\mathrm{O}$ atoms, the source of $10 \%$ of the oxygen in the coma, will give a $1 \%$ enhancement after two days. The $\mathrm{S} / \mathrm{N}$ of the Alice data do not permit such a detection. $\mathrm{CO}$ and $\mathrm{CO}_{2}$ are additional minor sources of oxygen atoms but have photodissociation lifetimes greater than 10 days at $1.5 \mathrm{AU}$ (Huebner et al. 1992), and so will not produce a detectable change.

\subsection{Limits on Carbon Monoxide}

Feldman et al. (2006) have modelled the images from the Solar Blind Channel of the HST/ACS to derive a quiescent $\mathrm{CO}$ production rate of $4-6 \times 10^{26}$ molecules s $^{-1}$ and a number of $1.5 \times 10^{31} \mathrm{CO}$ molecules produced by the impact. Modeling the upper value of $\mathrm{CO}$ quiescent outgassing in terms of radial outflow from the nucleus, we can estimate the expected CO Fourth positive emission in the central spatial pixel to be 0.024 rayleighs for the strongest $(1,0)$ and $(2,0)$ bands. The change in $\mathrm{CO}$ resulting from the impact will give an increase in brightness of 0.004 rayleighs in these bands, the low value being a consequence of the large FOV of the Alice slit and the concentration of the produced gas within a few thousand $\mathrm{km}$ of the nucleus. These values are well below the 3- $\sigma$ upper limits given in Table 1. Because of the long photodissociation lifetime of $\mathrm{CO}$, the atomic oxygen and carbon produced from $\mathrm{CO}$ will again give a negligible contribution to either the O I $\lambda 1304$ or C I $\lambda 1657$ brightness.

\section{CONCLUSION}

Observations of periodic comet 9P/Tempel 1 were made with the Alice ultraviolet spectrograph on the Rosetta spacecraft from six days before through ten days following the Deep Impact encounter with the comet. O I emission from the quiescent comet was clearly detected and an $\mathrm{H}_{2} \mathrm{O}$ production rate of $9 \times 10^{27}$ molecules $\mathrm{s}^{-1}$, with an estimated uncertainty 
of $\sim 30 \%$, was derived from the measured O I brightness profile. Any enhancement either immediately following the impact or in the subsequent ten days was less than $10 \%(1-\sigma)$. Our upper limits to the volatiles produced by the impact are consistent with estimates from other observations.

\section{ACKNOWLEDGMENTS}

We thank the ESA Rosetta Science Operations Centre (RSOC) and Mission Operations Center (RMOC) teams for their expert and dedicated help in planning and executing the Alice observations of comet Tempel 1. We also thank Claudia Alexander (JPL), Kristin Wirth (ESTEC), Detlef Koschny (ESTEC), Paolo Ferri (ESOC), Maarten Versteeg (SwRI), and Gerhard Schwehm (ESTEC) for their continued support of Alice. The work at Johns Hopkins University was supported by NASA's Jet Propulsion Laboratory through a subcontract from Southwest Research Institute.

\section{REFERENCES}

A'Hearn, M. F., Belton, M. J. S., Delamere, A., Blume, W. H. 2005a. Deep Impact: A Large-Scale Active Experiment on a Cometary Nucleus. Space Science Reviews 117, $1-21$.

A'Hearn, M. F., and 32 colleagues 2005b. Deep Impact: Excavating Comet Tempel 1. Science 310, 258-264.

Biver, N., and 16 colleagues 2005. Radio Observations Of Comet 9P/Tempel 1 Before And After Deep Impact. AAS/Division for Planetary Sciences Meeting Abstracts 37, 710.

Budzien, S. A., Festou, M. C., \& Feldman, P. D. 1994. Solar Flux Variability and the Lifetimes of Cometary $\mathrm{H}_{2} \mathrm{O}$ and $\mathrm{OH}$. Icarus 107, 164-188.

Combi, M. R., Harris, W. M., Smyth, W. H. 2004. Gas dynamics and kinetics in the cometary coma: theory and observations, in Comets II, ed. M. C. Festou, H. A. Weaver, \& H. U. Keller (Tucson: Univ. of Arizona), 523-552.

Dymond, K. F., Feldman, P. D., Woods, T. N. 1989. Observations of a Greenstein effect in the O I $1302 \AA$ emission of Comet Halley. Astrophysical Journal 338, 1115-1121.

Feldman, P. D., Cochran, A. L., Combi, M. R. 2004. Spectroscopic investigations of fragment species in the coma, in Comets II, ed. M. C. Festou, H. A. Weaver, \& H. U. Keller (Tucson: Univ. of Arizona), 425-447. 
Feldman, P. D., Lupu, R. E., McCandliss, S. R., Weaver, H. A., A'Hearn, M. F., Belton, M. J. S., Meech, K. J. 2006. Carbon Monoxide in Comet 9P/Tempel 1 before and after the Deep Impact Encounter. Astrophysical Journal 647, L61-L64.

Huebner, W. F., J. J. Keady, and S. P. Lyon 1992. Solar photo rates for planetary atmospheres and atmospheric pollutants. Dordrecht; Boston: Kluwer Academic.

Keller, H. U., and 11 colleagues 2005. Deep Impact Observations by OSIRIS Onboard the Rosetta Spacecraft. Science 310, 281-283.

Küppers, M., and 40 colleagues 2005. A large dust/ice ratio in the nucleus of comet 9P/Tempel 1. Nature 437, 987-990.

Lemaire, P., Emerich, C., Vial, J.-C., Curdt, W., Schühle, U., Wilhelm, K. 2002. Variation of the full Sun hydrogen Lyman $\alpha$ and $\beta$ profiles with the activity cycle. ESA SP-508: From Solar Min to Max: Half a Solar Cycle with SOHO 11, 219-222.

Meech, K. J., and 208 colleagues 2005. Deep Impact: Observations from a Worldwide EarthBased Campaign. Science 310, 265-269.

Mumma, M. J., and 13 colleagues 2005. Parent Volatiles in Comet 9P/Tempel 1: Before and After Impact. Science 310, 270-274.

Rottman, G., Woods, T., Snow, M., Detoma, G. 2001. The solar cycle variation in ultraviolet irradiance. Advances in Space Research 27, 1927-1932.

Schleicher, D. G., Barnes, K. L., Baugh, N. F. 2006. Photometry and Imaging Results for Comet 9P/Tempel 1 and Deep Impact: Gas Production Rates, Postimpact Light Curves, and Ejecta Plume Morphology. Astronomical Journal 131, 1130-1137.

Slater, D. C., Stern, S. A., Booker, T., Scherrer, J., A'Hearn, M. F., Bertaux, J.-L., Feldman, P. D., Festou, M. C., Siegmund, O. H. 2001. Radiometric and calibration performance results of the Rosetta UV imaging spectrometer ALICE. Proc. SPIE Vol. 4498, p. 239247, UV/EUV and Visible Space Instrumentation for Astronomy and Solar Physics, Oswald H. Siegmund; Silvano Fineschi; Mark A. Gummin; Eds. 4498, 239-247.

This preprint was prepared with the AAS LATEX macros v5.2. 
Table 1. 24-hour Averaged Upper Limits

\begin{tabular}{lcccccc}
\hline \hline & \multicolumn{5}{c}{ Days From Impact } \\
Feature & -1 to 0 & 0 to 1 & 1 to 2 & 2 to 3 & 3 to 4 \\
\hline CO 1510 $⿱ 亠 乂$ & $<0.83$ & $<0.53$ & $<0.66$ & $<0.54$ & $<0.79$ \\
C I 1561 $\AA$ & $<0.51$ & $<0.42$ & $<1.13$ & $<0.55$ & $<1.27$ \\
C I 1657 $\AA$ & $<0.86$ & $<1.21$ & $<1.19$ & $<1.00$ & $<1.00$ \\
\hline
\end{tabular}

Note. - Upper limits are 3- $\sigma$ and are given in rayleighs, extracted from detector row 15 using observations made in the central jailbar position. 


\section{FIGURE CAPTIONS}

Fig. 1.- Pre- and post-impact spectra from jailbar C. In both panels rows 15, 13, and 12 are shown, top to bottom, dispaced by 0.05 rayleighs $\AA^{-1}$ for clarity. Rows 13 and 12 are displaced $8.30 \times 10^{5} \mathrm{~km}$ and $1.24 \times 10^{6} \mathrm{~km}$ from the center of row 15 , respectively, projected on the sky. The total exposure times are 56,608 s (pre-impact) and 111,402 s (post-impact).

Fig. 2.- Spatial profiles of O I $\lambda 1304$ and H I Lyman- $\beta$ derived from the same data shown in Fig. 1. The observed Lyman- $\beta$ is primarily from interplanetary hydrogen rather than the comet. Statistical error bars $(1-\sigma)$ are shown. The result of a Haser model for an $\mathrm{H}_{2} \mathrm{O}$ production rate of $1 \times 10^{28}$ molecules $\mathrm{s}^{-1}$, with the nucleus centered in row 15 , is indicated by $\times$ in the pre-impact profiles.

Fig. 3.- Spatial profile of interplanetary H I Lyman- $\beta$ derived from the outer jailbars of the $\rho$ Leo observation. The total integration time is $5,800 \mathrm{~s}$. Statistical error bars $(1-\sigma)$ are shown. Rows 13 to 16 are contaminated by light from the star.

Fig. 4.- Daily average brightness of the observed O I $\lambda 1304$ emission. The data are the mean of rows 14 to 16 and the error bars are 1- $\sigma$ in the observed counts. 

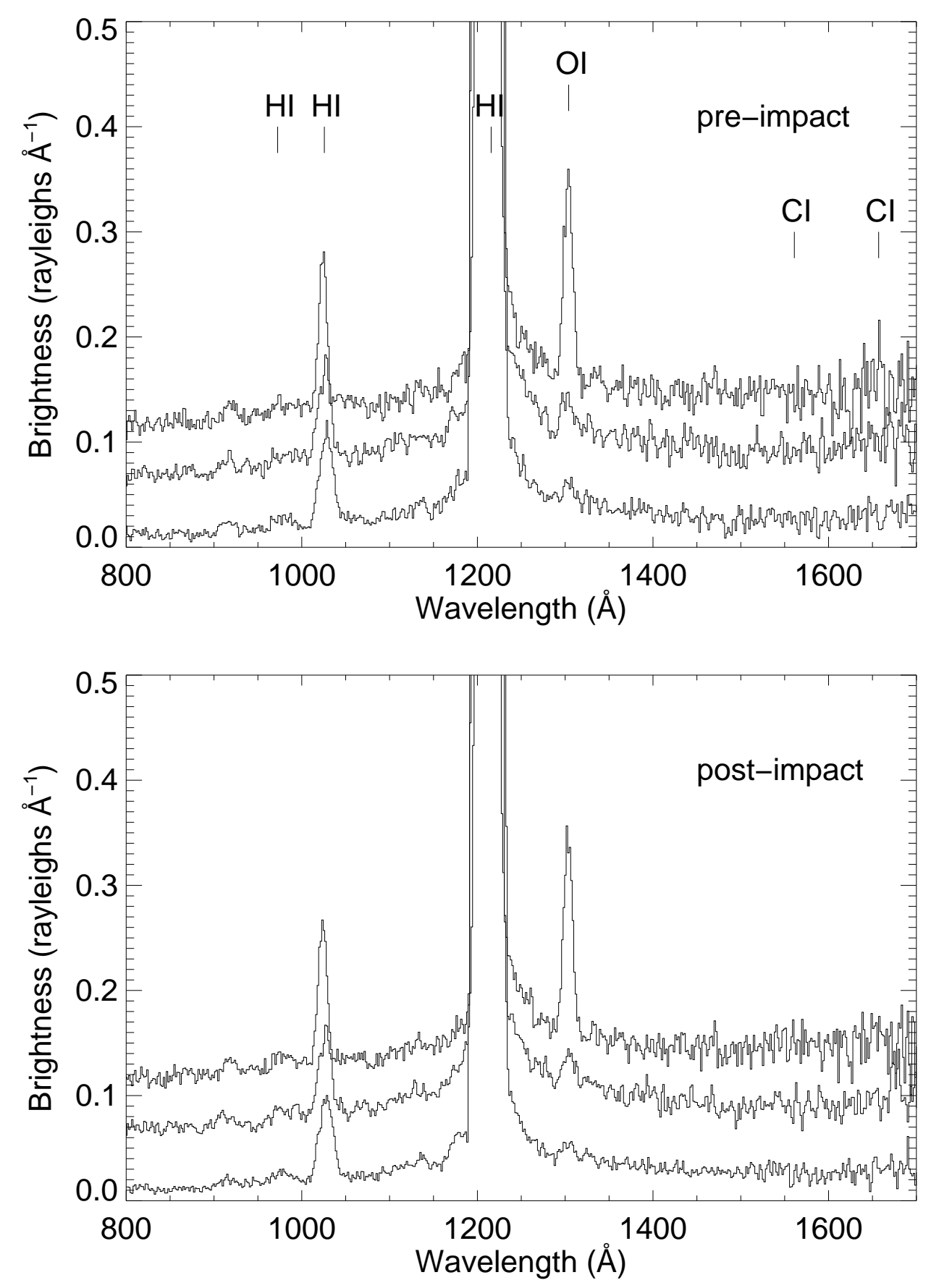

Fig. 1.- Pre- and post-impact spectra from jailbar C. In both panels rows 15, 13, and 12 are shown, top to bottom, dispaced by 0.05 rayleighs $\AA^{-1}$ for clarity. Rows 13 and 12 are displaced $8.30 \times 10^{5} \mathrm{~km}$ and $1.24 \times 10^{6} \mathrm{~km}$ from the center of row 15 , respectively, projected on the sky. The total exposure times are 56,608 s (pre-impact) and 111,402 s (post-impact). 

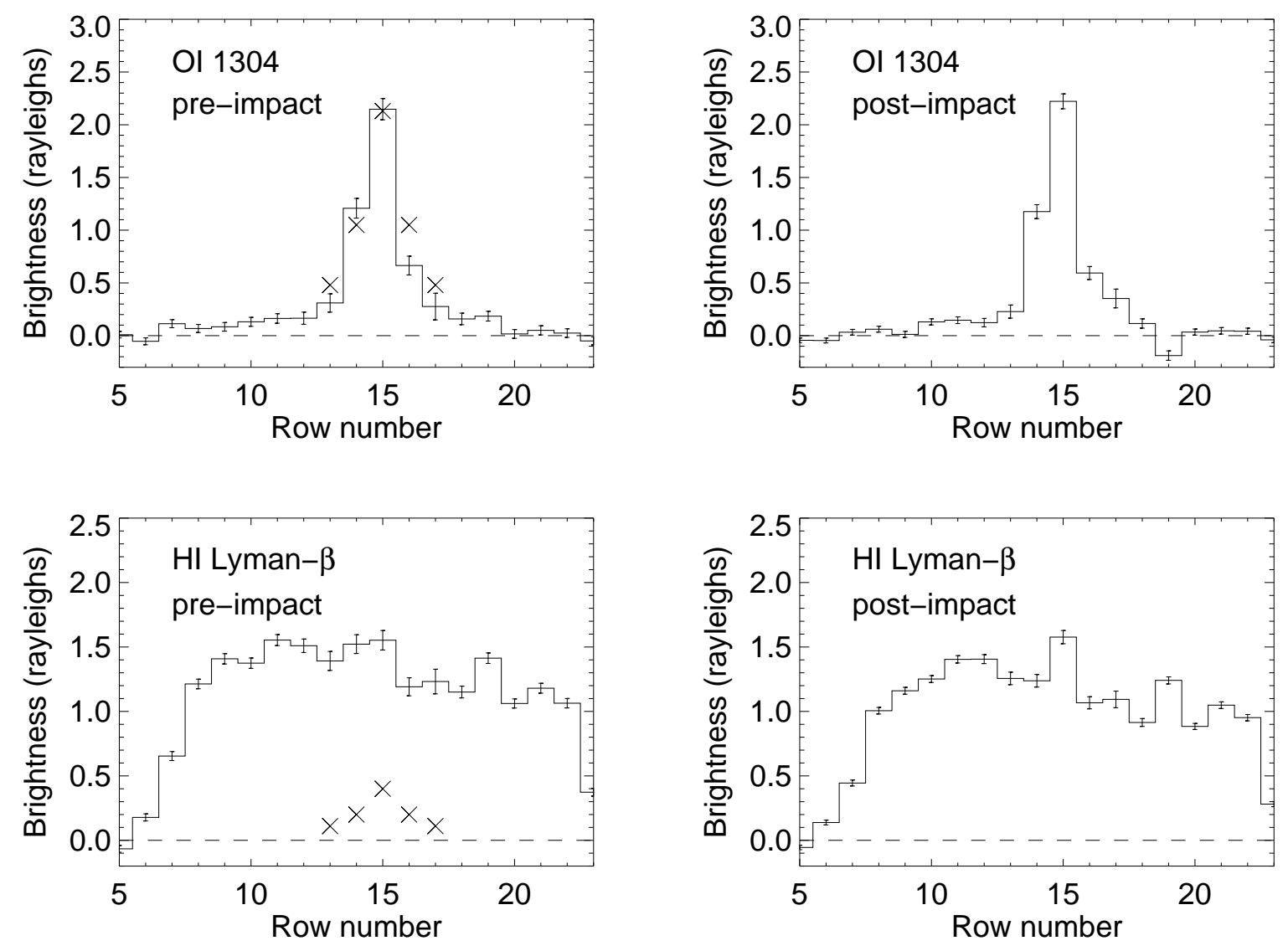

Fig. 2.- Spatial profiles of O I $\lambda 1304$ and H I Lyman- $\beta$ derived from the same data shown in Fig. 1. The observed Lyman- $\beta$ is primarily from interplanetary hydrogen rather than the comet. Statistical error bars $(1-\sigma)$ are shown. The result of a Haser model for an $\mathrm{H}_{2} \mathrm{O}$ production rate of $1 \times 10^{28}$ molecules $\mathrm{s}^{-1}$, with the nucleus centered in row 15 , is indicated by $\times$ in the pre-impact profiles. 


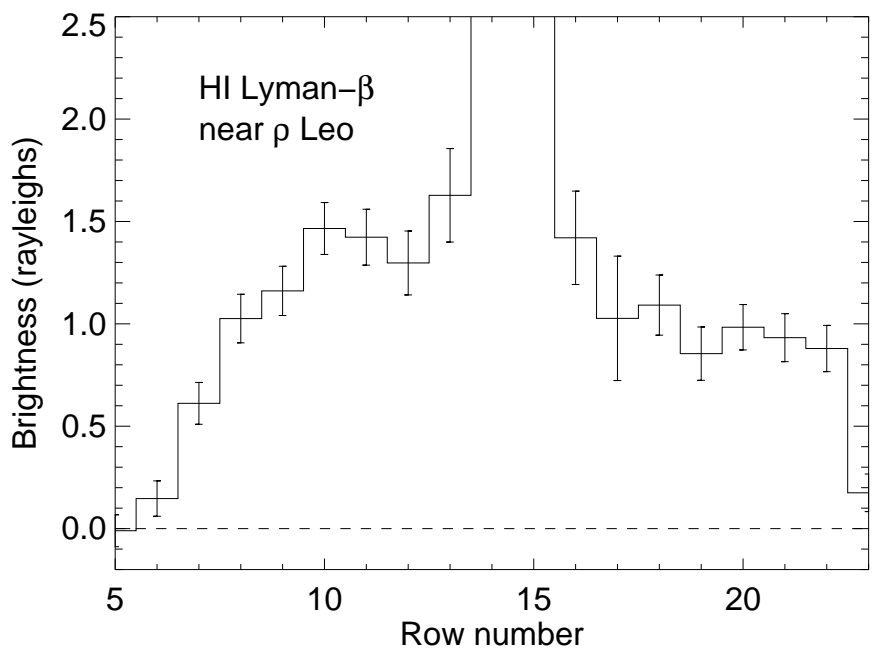

Fig. 3. - Spatial profile of interplanetary H I Lyman- $\beta$ derived from the outer jailbars of the $\rho$ Leo observation. The total integration time is $5,800 \mathrm{~s}$. Statistical error bars $(1-\sigma)$ are shown. Rows 13 to 16 are contaminated by light from the star. 


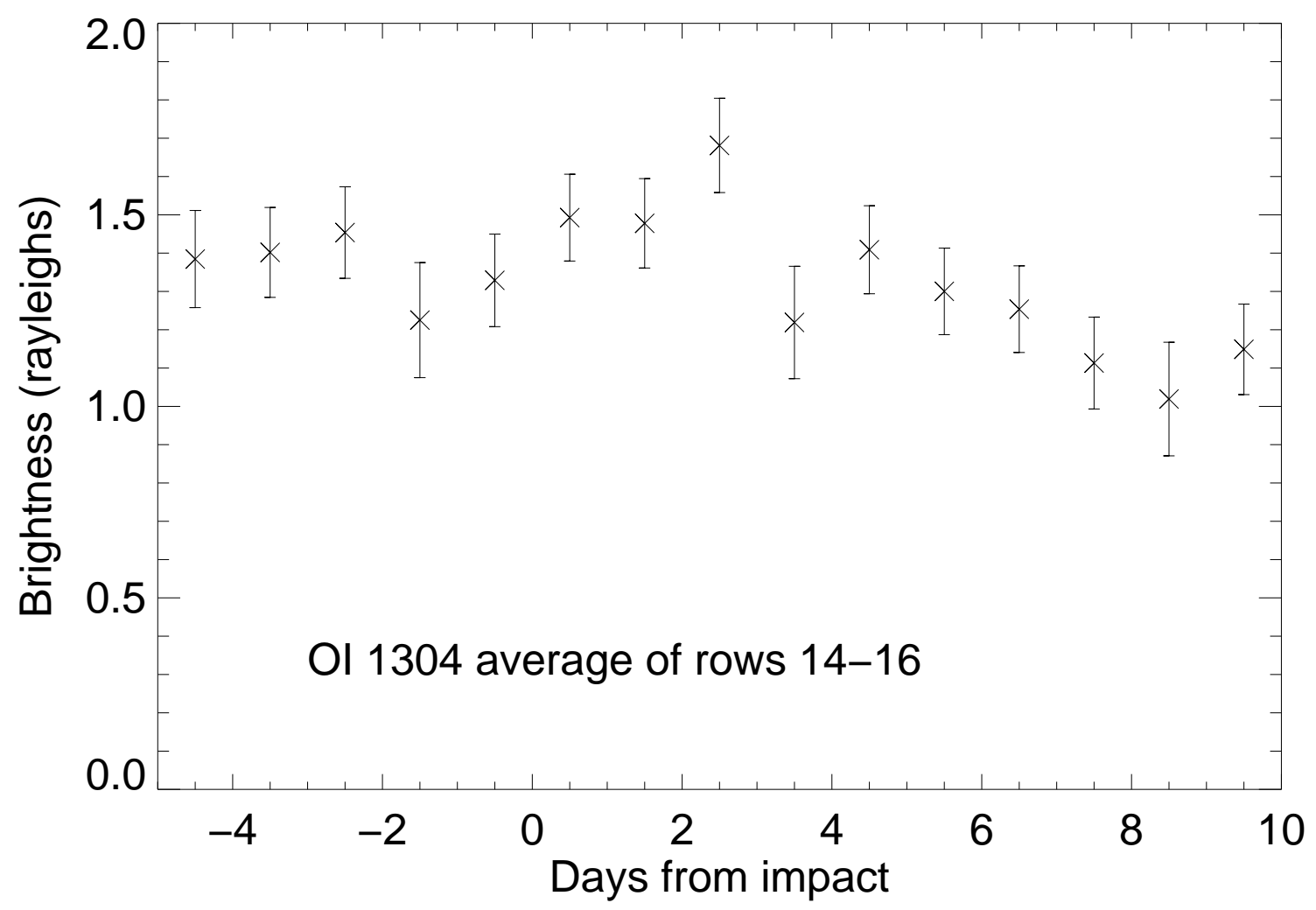

Fig. 4.- Daily average brightness of the observed O I $\lambda 1304$ emission. The data are the mean of rows 14 to 16 and the error bars are $1-\sigma$ in the observed counts. 